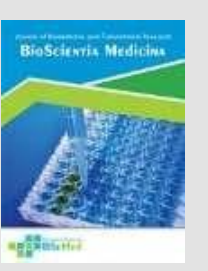

Bioscientia Medicina: Journal of Biomedicine \&

Translational Research

Journal Homepage: www.bioscmed.com

\title{
The Ethics of Gene Modification: Considerations of Gene Editing and Genetic Selection
}

\section{Murwani Emasrissa Latifah ${ }^{1^{*}}$}

${ }^{1}$ Obstetric And Gynecology Department, Mohammad Hoesin General Hospital, Palembang, Indonesia

\author{
A R T I C L E I N F O \\ Keywords: \\ Ethics \\ Gene Modification \\ Gene Editing \\ Gene Selection

\section{*Corresponding author:} \\ Murwani Emasrissa Latifah \\ E-mail address: \\ rissa.latifah@yahoo.co.id
}

\begin{abstract}
A B S T R A C T
The field of hereditary qualities has offered ascend to innovation that will reform the natural sciences: the amazing quality altering device known as CRISPR-Cas9. Between clinical advancement, extreme new age solution for sicknesses and the danger of modifying human DNA in an irreversible manner, the lawful difficulties of applying CRISPR in the wellbeing setting, alongside the administrative and moral issues that may emerge should be drawn nearer with expanded duty. A primary objective or an assortment of purposes? Upgrade or quality altering to improve ordinary human characteristics, changes from altered genomes that may be acquired - repercussions of the new quality altering innovation could bring about adjusting human DNA. Current worldwide enactment and understanding, worldwide agreement worth seeking after regarding this matter and future guidelines required.
\end{abstract}

All authors have reviewed and approved the final version of the manuscript.

https://doi.org/10.32539/bsm.v5i5.235

\section{Introduction}

The label 'genome editing technologies' commonly refers to technologies that allow scientists to form changes within the genetic sequences of organisms. Current genome editing technologies include zincfinger nucleases (ZFNs), transcription activator-like effector-based nucleases (TALENs) and clustered regularly interspaced short palindromic repeats (CRISPR), with CRISPR-associated nucleases [1]. CRISPR-based genome editing is taken into account more precise (it is feasible to focus on specific sequences of DNA), more efficient (it has relatively few off-target effects) and cheaper to use than other genome editing [2].

As momentum builds around CRISPR's experimental uses, a considerable debate has developed amongst scholars from a good range of disciplines [1-2], national academies, ethics [3-4], members of the general [5-6], learned and patients [8].

This debate concerns the moral acceptability of its human applications, among others, and therefore the mechanisms of governance that might be needed to manage these applications. CRISPR is an emerging biotechnology, and lots of view it as having a high level of disruptive potential for biomedical research and its associated ethical landscape [8-11].

Disruption could occur by making previously inefficient procedures more efficient, by making hypothetical procedures possible, or by allowing new processes to be conceived [13]. The rapidity by which CRISPR has been adopted as an experimental 
technique in laboratories and as a gene transfer technology is partial evidence to a number of this disruptive [10].

\section{Gene editing}

Counterfeit control of qualities is a moderately new science, and various turning points have given the establishment to the present status of hereditary designing. Analysts first discovered that vague changes to Drosophila DNA could be introduction induced utilizing radiation and chemicals in 1927 and 1947 [14]. More prominent comprehension of the design of the DNA particle, (for example, crafted by Watson, Crick, and Franklin, prompting the discovery of DNA's twofold helix structure) and the cell measures that administer its record, interpretation, replication, and fix, (for example, the capacity of ligases and limitation enzymes) prompted the primary joining experiments and, eventually, the principal recombinant DNA7 in the mid 1970s [15].

DNA recombination procedures were utilized extensively in the sprouting yeast Saccharomyces cerevisiae, starting in the mid 1980s, permitting scientists to contemplate utilitarian eukaryotic genomics. What's more, in a critical progression, the improvement of polymerase chain response (PCR) permitted researchers to intensify DNA, delivering a great many duplicates from a solitary strand [14].

Around a similar time, various labs made the first transgenic mice and around five years after the fact, the primary knockout mice were created. Targeted quality altering was additionally cutting-edge by the disclosure that designed endonucleases could make site-explicit twofold abandoned breaks (DSBs), which thusly actuate homologous recombination (HR) the most wellknown kind of homology-directed fix (HDR). At the point when the Human Genome Project was declared complete in 2003, it got conceivable to distinguish (and consequently, hypothetically, focus on) any human quality of interest[14].

The three fundamental methods for quality altering include particles that perceive and tie to explicit DNA successions; researchers can utilize custom atoms to influence hereditary and epigenetic changes on basically any quality. For instance, these atoms can be joined with endonucleases, making DSBs which can be fixed utilizing either nonhomologous end joining (NHEJ), which regularly brings about little arbitrary indel transformations, or HDR, which, when benefactor DNA with homology to one or the other side of the cleavage site is available, can be utilized to make new or "fixed" adaptations of an objective quality. The siteexplicit DNA acknowledgment atom can likewise be joined with an effector particle to up-or downregulate quality articulation.

\section{ZFPs/ZFNs}

In the last part of the 1970s and mid 1980s, there was an enormous spotlight on understanding record factor IIIA (TFIIIA), the main eukaryotic transcription factor to be portrayed. In 1983, specialists established that zinc is needed for TFIIIA function and in 1985 came the disclosure that the zinc-restricting bits of the proteins are really rehashing themes, autonomously collapsed to make finger-like areas that hold the DNA. This class of proteins is presently alluded to as zinc finger proteins (ZFPs), and a few comparative proteins have been discovered in the proteomes of various creatures. Since each zinc finger perceives three base pairs, a peptide can be made to perceive an objective quality by joining the proper zinc fingers in a direct design [14-15].

A 1994 paper portrays a ZFP that was designed to perceive and smother an oncogene, just as a ZFP that acted (in an alternate cell framework) as an advertiser of another quality by perceiving its activation domain a similar paper recommends that ZFPs can be bound to effector proteins as a methods for controlling quality articulation. Expanding on this thought, analysts combined a ZFP to the vague cleavage space of the Fok1 limitation enzyme. The subsequent heterodimer, known as a zinc finger nuclease $(\mathrm{ZFN})$, can perceive a particular DNA succession and produce a focused on DSB. As recently referenced, these DSB can either be fixed through NHEJ, bringing about little indels, or HDR, which can be outfit to embed a substitute or fixed quality. Fok1 must dimerize, so ZFNs should be made two by two (one focusing on the 3' strand and the other 
focusing on the $5^{\prime}$ strand) which improves target particularity-however productivity remains moderately low (G-rich groupings are particularly hard to target)[14-15].

Ex vivo and in vivo conveyance of ZFNs is generally simple given their little size and the little size of the ZFN tapes (which considers the utilization of an assortment of vectors). Be that as it may, while ZFNs were absolutely novel at the time they were created, they are staggeringly troublesome and costly to design, making them less functional when all is said in done than more up to date innovations[14].

\section{TALES/TALENs}

In 2009, two unique research facilities depicted a recently distinguished DNA-restricting theme: the record activator-like effector (TALE), a protein emitted by the plant microbe Xanthomonas. Each TALE incorporates a DNA-restricting area made out of couple rehashes with rehash variable diresidue (RVDs) at positions 12 and 13; each RVD perceives an individual nucleotide [14].

Like ZFPs, manufactured TALEs can be intended to influence quality regulation, joined with effector proteins, or intertwined to endonucle-ases26-28 to make TALE nucleases (TALENs); likewise with ZFNs, in light of the fact that Fok1 is the endonuclease utilized, TALENs should be made two by two [14].

Story nucleases are a lot bigger than ZFNs, thus can be more hard to convey effectively (particularly in vivo). Notwithstanding, for some reasons (counting the idea of their relative communications with the DNA and the way that each RVD perceives a solitary base), TALEbased delusions (particularly TALENs) can be worked with higher explicitness and more prominent focusing on limit than ZFP-based figments. Moreover, TALENs can be created altogether more inexpensively, easily, and with more noteworthy proficiency than ZFNs [14].

\section{CRISPR-Cas}

In 1987, a research center in Osaka inadvertently found an unusual palindromic rehash arrangement in the E. coli genome they were contemplating, one of a kind in that it was consistently interspaced. These DNA themes were additionally recognized in different bacterial genomes by various labs over the course of the following 20 years; their capacity, how-ever, was as yet unclear. By 2005, three gatherings had freely discovered that the spacer groupings were really gotten from phage DNA and the chance of the qualities assuming a part in bacterial invulnerability was first suggested [14]. By this time, the scientific local area alluded to this uncommon exhibit as bunched regularly interspaced short palindromic rehashes, or CRISPR. Then, analysts in the Netherlands had recognized a few different qualities situated close to the CRISPR locus that gave off an impression of being practically associated with the CRISPR genes, these would end up being the CRISPR related proteins (Cas) that make up a fundamental piece of the CRISPR-Cas framework[14].

In 2007, the CRISPR-Cas framework was distinguished just like a supportive of karyotic safeguard against pathogens. As a piece of a self-/nonself-assurance system of versatile invulnerability, prokaryotes coordinate a section (for the most part 3238 base sets) of phage DNA into their own genome, making the spacers in the CRISPR clusters. After the CRISPR qualities are translated, endoribonucleases divide the subsequent CRISPR RNA (pre-crRNA), bringing about more limited RNA units made out of a solitary spacer succession and the palindromic rehash (crRNA); contingent upon the living being, a transenacting crRNA (tra-crRNA) may likewise be deciphered. The RNA frames a ribonucleoprotein (RNP) complex with the related Cas proteins; any phage DNA containing the spacer succession will be distinguished by the controlling RNA and cut by the endonuclease capacity of the Cas protein(s). The protospacer is the homologous grouping in the attacking DNA, and is trailed by a short protospacer neighboring theme (PAM); in light of the fact that the PAM isn't fused in the CRISPR cluster, the CRISPR-Cas complex can perceive the unfamiliar DNA as non-self (and accordingly won't cut the prokaryotic cell's own DNA) [14].

In 2012, Jennifer Doudna, Emmanuelle Charpentier, and others in their group designed a manufactured delusion of the tracrRNA and crRNA (presently known as single guide RNA, or sgRNA), 
which had the option to coordinate Cas9 to make a focused, available explicit twofold abandoned break.37 By 2013, specialists had set up that the CRISPR-Cas9 was a viable, easy, and multiplexable technique for altering the human genome[16].

By and large, nonetheless, CRISPR is for the most part the preferred technique for hereditary and epigenetic control, particularly as upgrades are made to the innovation. CRISPR's principle advantage over its archetypes lies in the way that as opposed to a mind boggling protein as the DNA acknowledgment particle, the CRISPR framework depends on a guide RNA. CRISPR units are hence altogether less expensive, simpler, and more proficiently delivered than either ZFNs or TALENs[11].

\section{Gene selection}

Hereditary determination occurs in nature common choice is the mechanism that drives Darwinian development. People have likewise been rehearsing fake determination for millennia, choosing for phenotypic qualities when rearing plants and creatures. New technologies have been created throughout the most recent 53 years that permit selection of an undeveloped organism dependent on different measures, for example, sex, ploidy, and polymorphisms [17].

Preimplantation hereditary testing (PGT) envelops different techniques used to screen undeveloped organisms preceding exchange. Initially all referred to as preimplantation hereditary analysis (PGD), there are really three sorts of PGT: aneuploidy location, presently called PGT-A; monogenic confusion discovery, presently called PGT-M; and primary revision recognition, presently called PGT-SR. Regularly, IVF isn't possible, requiring post-implantation pre-birth testing (when shown by family ancestry and other danger factors). Amniocentesis, chorionic villus examining (CVS), and percutaneous umbilical line inspecting (PUBS) were at first combined with karyotyping, which can identify sex, aneuploidy, and a few sorts of underlying chromosomal issues. Karyotyping was supplanted by chromosomal microarray strategies (aCGH and SNP exhibit) and, more recently, low-pass genome sequencing, as these innovations permit discovery of CNVs just as aneuploidy[17].

\section{Ethical issues in somatic genome editing}

The IBC hence required a ban on this particular system, due to many issue relating to the ethical of genome editing. At its gathering, on the human genome and common liberties. Ongoing advances have made the way for hereditary screening and testing for acquired infections, quality treatment, the utilization of undeveloped undifferentiated organisms in clinical exploration and the chance of cloning and hereditary "altering" for both clinical and non-clinical closures. Mediations on the human genome ought to be conceded distinctly for preventive, symptomatic or restorative reasons and without authorizing alterations for relatives.

Another genome "altering" method called CRISPRCas9 makes it feasible for researchers to embed, eliminate and right DNA just and eficiently. It holds out the possibility of treating or in any event, restoring certain sicknesses, for example, sickle cell illnesses, cystic fibrosis and a few malignancies. In any case, germline altering can likewise make changes to DNA, for example, deciding an infant's eye tone, simpler for researchers working with human undeveloped organisms, eggs and sperm[18].

Despite the fact that cases of the expected advantages of GGE regularly identify with the prospects of relieving, or forestalling infection, the genuine bit of leeway of GGE over accessible techniques is questionable and at present seem restricted. Initially, GGE doesn't have remedial points fundamentally since there is no patient associated with the strategy who could be cured1. All things being equal, GGE in the facility would be combined with the in vitro preparation (IVF) method to make a hereditarily related youngster (for a given couple) and who might then have an ideal quality (for example would not be influenced by a given infection) [19].

This methodology would be offered in the principal case to couples who have a blend of genotypes that will result (from a certain perspective) in a portion of their youngsters being influenced by a hereditary dis-1 In 
one of the methodologies of GGE, the incipient organism can be considered as the subject which could be relieved, that is, when GGE is applied on a current incipient organism. This methodology, notwithstanding, is known to cause mosaicism in undeveloped organisms, and accordingly, is probably not going to be genuinely considered for clinical employments. The strategy for adding the parts of a GE framework right now of preparation appears to be more beneficial as far as wellbeing of possible clinical employments[19].

Straight forwardness and who know about this and might want to dodge giving dis-124 simplicity to their posterity. Significantly, such couples immediately have 125 various alternatives accessible to accomplish the perfect objective: 1) 126 undergo IVF combined with preimplantation hereditary analysis 127 (PGD) to line tle on "unaffected" undeveloped organisms and use them to set up 128 pregnancy; PGD can identify post-IVF undeveloped organisms, which convey 129 sickness causing alleles and considers the selection of incipient organisms 130 without these (mixes of) alleles; 2) use giver gametes in 131 IVF; 3) receive a youngster; 4) prefer to not have a child together[19].

In most of cases, IVF combined with PGD are often applied and GGE doesn't appear to possess an unmistakable little bit of leeway over it as we clarify within the section beneath. However, from a certain point of view, there are uncommon situations where all offspring of a couple would be influenced by an infection (for instance, when one parent is homozygous for a prevailing dis-simplicity, or the two guardians are homozygous for a latent change) and there is no choice for PGD and incipient organism choice[19].

In such situations, GGE may conceivably be the simply choice to have a genetically related youngster and, all things considered, it could bring a bit of leeway more than right now accessible strategies. Significantly, it isn't certain whether such couples exist and in the event that they do exist, regardless of whether they would be willing to go through GGE. Without a doubt, the hypothetical assessment dependent on accessible information on pervasiveness of hereditary issues in the USA proposes that the clinical interest for GGE would be minuscule [16]. For model, the investigation demonstrates that in the USA in a given time there is just one couple at regenerative age in which the two people are homozygous for variations causing cystic fibrosis[20].

\section{Conclusion}

In the pursuit to pushing moral limits in the journey for more noteworthy great, before CRISPR can be generally acknowledged and utilized, an expanded degree of wellbeing that grants clinical applications in the short term is completely required. There are a few basic difficulties and future possibilities of CRISPRbased frameworks for human examination yet in addition the enactment worldwide should stay aware of clinical advancement, control and ensure every basic liberty. Every single nation should develop instruments of administration for managing research with genome altering in people and should consider whether it ought to be allowed concerning socioethical suggestions just as wellbeing and viability. Likewise, preventive measures against manhandles should be mulled over, just as a worldwide collaboration and agreement, since the domino impact may include the whole mankind.

Despite the fact that it's probably not going to wipe out all dangers, it is seemingly one of only a handful few alternatives accessible to sensibly control and additionally limit them.

Without a doubt, it is critical to manage moral issues brought by quick switches up in medication, life sciences and innovation. Human genome is important for the legacy of mankind. It subsequently plots decides that should be seen to regard human nobility, basic liberties and basic opportunities.

\section{Acknowledgment}

We express our gratitude to the researcher who had been invented this genome modification in good purpose, we hope this manuscript may contribute to the ethical consideration regarding gene modification uses. 


\section{References}

[1] W. H. Organization, "WHO Expert Advisory Committee on Developing Global Standards for Governance and Oversight of Human Genome Editing." WHO. http://www. who. int/ethics/topics/humangenomeediting/committee-members/en, 2019.

[2] K. S. Bosley et al., "CRISPR germline engineering - The community speaks," Nature Biotechnology. 2015, doi: 10.1038/nbt.3227.

[3] M. J. Legato et al., "Editing the Human Genome: Progress and Controversies," Gend. Genome, 2017, doi: 10.1089/gg.2016.29001.rt1.

[4] "Genome Editing - An Ethical Review," Jahrb. für Wiss. und Ethik, 2018, doi: 10.1515/jwiet2017-0014.

[5] Nuffield Council on Bioethics, Genome editing and human reproduction. 2018.

[6] G. Gaskell et al., "Public views on gene editing and its uses," Nature Biotechnology. 2017, doi: 10.1038/nbt.3958.

[7] D. A. Scheufele, M. A. Xenos, E. L. Howell, K. M. Rose, D. Brossard, and B. W. Hardy, "U.S. attitudes on human genome editing," Science. 2017, doi: 10.1126/science.aan3708.

[8] A. van Mil, H. Hopkins, and S. Kinsella, "Potential uses for genetic technologies: dialogue and engagement research conducted on behalf of the Royal Society," R. Soc., 2017.

[9] H. Ledford, "CRISPR, the disruptor," Nature, 2015, doi: 10.1038/522020a.

[10] M. Braun and P. Dabrock, "Mind the gaps!: Towards an ethical framework for genome editing," EMBO Rep., 2018, doi: 10.15252/embr.201745542.

[11] C. Mariscal and A. Petropanagos, "CRISPR as a driving force: the Model T of biotechnology," Monash Bioeth. Rev., 2016, doi: 10.1007/s40592-016-0062-2.
[12] M. Schultz-Bergin, "Is CRISPR an Ethical Game Changer?," J. Agric. Environ. Ethics, 2018, doi: 10.1007/s 10806-018-9721-z.

[13] S. H. Khan, "Genome-Editing Technologies: Concept, Pros, and Cons of Various GenomeEditing Techniques and Bioethical Concerns for Clinical Application," Molecular Therapy Nucleic Acids. 2019, doi: 10.1016/j.omtn.2019.02.027.

[14] J. Rothschild, "Ethical considerations of gene editing and genetic selection," J. Gen. Fam. Med., 2020, doi: 10.1002/jgf2.321.

[15] J. D. Watson and F. H. C. Crick, "Molecular structure of nucleic acids: A Structure for deoxyribose nucleic acid," in 50 Years of DNA, 2016.

[16] M. Jinek, K. Chylinski, I. Fonfara, M. Hauer, J. A. Doudna, and E. Charpentier, "A programmable dual-RNA-guided DNA endonuclease in adaptive bacterial immunity," Science (80-. )., 2012, doi: $10.1126 /$ science. 1225829.

[17] H. Wang et al., "Low-pass genome sequencing versus chromosomal microarray analysis: implementation in prenatal diagnosis," Genet. Med., 2020, doi: 10.1038/s41436-019-0634-7.

[18] ONU - UNESCO, "Panel of experts calls for ban on 'editing' of human DNA to avoid unethical tampering with hereditary traits," UNESCO, 2015. .

[19] E. Niemiec and H. C. Howard, "Ethical issues related to research on genome editing in human embryos," Comput. Struct. Biotechnol. J., vol. 18, pp. 887-896, 2020.

[20] M. Darnovsky, "Genetically modifying future children isn't just wrong: It would harm all of us," Guard., 2018. 